\section{ralud mental}

Flores-Gutiérrez, Enrique Octavio; Cervantes, Juan José; Torres Álvarez, Marina; Alcauter Solórzano, Sarael

Procesamiento de la música en el primer episodio de trastorno depresivo mayor sin tratamiento

Salud Mental, vol. 36, núm. 6, noviembre-diciembre, 2013, pp. 449-457

Instituto Nacional de Psiquiatría Ramón de la Fuente Muñiz

Distrito Federal, México
Salud Mental

Instituto Nacional de Psiquiatría Ramón

de la Fuente Muñiz

México revistasaludmental@imp.edu.mx

Disponible en: http://www.redalyc.org/articulo.oa?id=58229682002

- Cómo citar el artículo

- Número completo

- Más información del artículo

- Página de la revista en redalyc.org 


\title{
Procesamiento de la música en el primer episodio de trastorno depresivo mayor sin tratamiento
}

\author{
Enrique Octavio Flores-Gutiérrez, ${ }^{1}$ Juan José Cervantes, ${ }^{1}$ Marina Torres Álvarez, ${ }^{2}$ Sarael Alcauter Solórzano ${ }^{3}$
}

Artículo original

\section{SUMMARY}

The purpose of this study is the assessment of the differences in brain activity when patients with major depressive disorder (MDD) listen to two different types of music, with healthy subjects as control, by using functional magnetic resonance imaging (fMRI). Brain activity in musical stimuli with healthy subjects has been investigated extensively, but there are few neurobiologic music studies in mental illness, particularly in MDD. Studies in this area provide a new perspective on interdisciplinary research to explore the neurobiological substrates of MDD. This study involved 20 male subjects: 10 patients $(34 \pm 7$ years), and 10 control subjects ( $33 \pm 7$ years). The MDD Patients were selected in the pre-consultation service of the National Institute of Psychiatry Ramón de la Fuente Muñiz (INPRFM) of Mexico City, and control subjects were selected among workers of the Institute who responded to the invitation. All participants completed the Hamilton scales for anxiety and depression, Beck inventories for depression and anxiety and, the SCL-90-R. The Mini-Mental State Examination test was also administered to patients for diagnostic purposes. The fMRI was obtained by Philips Achieva 3-Tesla in the INPRF; the analysis was done using SPM2 format MRIcro system. The experimental stimuli were two pieces of music: one by JS Bach validated as quiet and another one by J Prodromidès validated as disturbing. Results show differences between both groups of subjects and between types of music. In all cases, the parahippocampal area, the tail of the caudate nucleus and the auditory temporal cortex were activated. The neurobiological processing of music is affected by MDD. We discuss the clinical and cognitive implications of these findings.

Key words: Major depressive disorder, functional magnetic resonance imaging, music.

\section{RESUMEN}

El propósito de este estudio fue registrar diferencias durante la audición de dos tipos diferentes de música en pacientes con Trastorno Depresivo Mayor (TDM), comparados con sujetos sanos, mediante imagen por resonancia magnética funcional (IRMf). La actividad cerebral con estímulos musicales ha sido investigada ampliamente en sujetos sanos, pero son escasos los estudios del procesamiento de la música en estados de patología mental, particularmente en el TDM. Los estudios en esta área interdisciplinaria proveen una nueva perspectiva de investigación para explorar los sustratos neurobiológicos del TDM. Participaron 20 sujetos de sexo masculino: 10 pacientes con TDM ( $34 \pm 7$ años) y 10 sujetos control (33 \pm 7 años). Los pacientes se seleccionaron en el servicio de pre-consulta del Instituto Nacional de Psiquiatría Ramón de la Fuente Muñiz (INPRFM) de la Ciudad de México, y los sujetos control entre los trabajadores del propio Instituto que respondieron a la invitación. Todos los participantes contestaron, con fines de confirmar el diagnóstico, las escalas de ansiedad y depresión de Hamilton, los inventarios de Beck para ansiedad y depresión y el SCL-90-R. A los pacientes se les aplicó además el MINI-mental test. Para la IRMf se usó un equipo Philips Achieva de tres Teslas en el INPRFM, el análisis se hizo con el formato SPM2 usando el sistema MRIcro. Los estímulos experimentales fueron una obra musical de JS Bach validada como tranquila y otra de J Prodromidès validada como inquietante. Los resultados muestran diferencias tanto entre los grupos de sujetos como entre los tipos de música: en todos los casos se activó el área parahipocampal, la cola del núcleo caudado y la corteza temporal auditiva. Concluimos que el procesamiento neurobiológico de la música es afectado por el TDM. Se discuten las implicaciones clínicas y cognoscitivas de estos hallazgos.

Palabras clave: Trastorno depresivo mayor, imagen por resonancia magnética funcional, música.

\section{INTRODUCCIÓN}

El TDM tiene serias repercusiones sobre la calidad de vida y el funcionamiento social de los pacientes afectados. En
México tiene una alta tasa de morbilidad al grado de ser causa de importantes niveles de ausentismo en el trabajo., ${ }^{1,2}$ La Organización Mundial de la Salud reportó que la depresión podría ser, para el 2020, la segunda causa de deterioro

\footnotetext{
Dirección de Investigaciones Clínicas. Instituto Nacional de Psiquiatría Ramón de la Fuente Muñiz.

Dirección de Servicios Clínicos INPRFM.

Instituto de Neurobiología, UNAM.
}

Correspondencia: Enrique O. Flores-Gutiérrez. Dirección de Investigaciones Clínicas. Instituto Nacional de Psiquiatría Ramón de la Fuente Muñiz. Calz. MéxicoXochimilco 101, San Lorenzo Huipulco, Tlalpan, 14370 México, DF. E.mail: florese@imp.edu.mx

Recibido: 29 de enero de 2013. Aceptado: 16 de julio de 2013. 
en el funcionamiento social y laboral en el mundo. Además, en los últimos años la incidencia de este trastorno aumentó no sólo en los adultos sino también, de forma alarmante, en niños y jóvenes. ${ }^{3}$ Ante estas expectativas es importante desarrollar investigaciones que aporten nuevos conocimientos sobre este trastorno y estrategias para el tratamiento del paciente afectado.

La relación del trastorno depresivo con la música tiene antecedentes en la literatura científica y se ha abordado desde la clínica, aunque son pocas las publicaciones relacionadas Por ejemplo, Nielzén y Cesarec $^{4}$ comparan pacientes con varias patologías psiquiátricas y sujetos control (s/C), y encuentran que la experiencia musical en los pacientes deprimidos resulta menos gratificante que para los s/C y que para los otros pacientes con diferentes diagnósticos psiquiátricos. Otro estudio reciente, el de Punkanen et al., ${ }^{5}$ compara pacientes con depresión y un grupo de s/C expuestos a estímulos musicales. Estos autores demuestran un sesgo emocional negativo en los pacientes con depresión y concluyen que la evaluación de las emociones musicales podría ser un medio pre-diagnóstico para deducir la presencia de depresión. Otro estudio más, realizado por Naranjo et al., ${ }^{6}$ confirma que el procesamiento de estímulos emocionales producidos por la música en el TDM se asocia a un "sesgo negativo general" y concluyen que la alteración del procesamiento emocional no se confina a estímulos interpersonales (caras y voces), sino que también se manifiesta en la incapacidad de experimentar la música normalmente. Por último, Osuch et al., ${ }^{7}$ hicieron un estudio de imagen cerebral con IRMf en pacientes con depresión, comparándolos con s/C. Al escuchar temas musicales seleccionados por los propios participantes como favoritos contra temas neutros, reportaron respuestas en el circuito de recompensa de los s/C y déficits significativos en los pacientes depresivos.

Estos trabajos nos sugieren que la respuesta a los estímulos musicales puede ser útil para hacer evaluaciones afectivas, cognoscitivas y fisiológicas en tiempo real, que podrían tener posibles aplicaciones preventivas, diagnósticas, de tratamiento y de rehabilitación en la clínica, dado que son estímulos fácilmente administrados, y cada vez más se difunde el sustento clínico y la evidencia neurobiológica de su eficacia..$^{6-8}$

En el presente estudio planteamos que al escuchar música, la participación cerebral del sistema afectivo en los p/ TDM comparados con los s/C tendrá diferencias apreciables con la técnica de IRMf. Se usaron estímulos opuestos en su efecto emocional para contrastar su consecuencia en el procesamiento cerebral.

Reclutamos pacientes en su primer episodio y sin tratamiento previo ya que se han reportado cambios neurofisiológicos y cognitivos variables en la depresión recurrente a diferencia de los pacientes con un primer episodio., ${ }^{9,10}$ Por otra parte los pacientes con tratamiento antidepresivo o con consumo previo de otras drogas pueden presentar diferencias importantes en los estudios de imagen cerebral al ser comparados con pacientes sin tratamiento previo. ${ }^{11-13}$ Además, a pesar de que el TDM se presenta dos veces más en las mujeres que en los varones, decidimos realizar este proyecto únicamente con sujetos de sexo masculino dado que en las mujeres hay variaciones hormonales periódicas que pueden alterar la percepción de los estímulos afecti$\operatorname{vos}^{14}$

\section{MÉTODO}

\section{Sujetos}

Participaron 20 varones adultos de entre 21 y 45 años de edad, diestros y no músicos. Diez fueron los sujetos control, clínicamente sanos (33 \pm 7 años), trabajadores del Instituto Nacional de Psiquiatría Ramón de la Fuente que respondieron a la invitación para participar, comparados con los pacientes por el método de pares. Otros diez fueron pacientes con diagnóstico primario de TDM, en su primer episodio y sin tratamiento (34 \pm 7 años), reclutados por medio del Servicio de pre-consulta de los Servicios clínicos del mismo Instituto, seleccionados de acuerdo a los criterios diagnósticos para trastorno depresivo mayor según el DSM-IV-TR. Además, los participantes debieron cumplir con las siguientes condiciones:

- Pacientes. Criterios de inclusión: hombres, diestros y no músicos, con edad de entre 21 y 45 años, con el diagnóstico de trastorno depresivo mayor según el DSM-IV y el MINI-mental Test, y con una puntuación igual o mayor a 22 puntos en la escala de depresión de Hamilton, y no haber recibido tratamiento farmacológico psiquiátrico previo. Criterios de exclusión: enfermedad médica descompensada, ideación o intento suicida reciente, depresión grave con síntomas psicóticos, comorbilidad con otros trastornos psiquiátricos, abuso o dependencia a sustancias adictivas.

- $\quad$ Sujetos control. Criterios de inclusión: Hombres diestros y no músicos, con edad de entre 21 y 45 años, no presentar algún diagnóstico psiquiátrico ni haber recibido tratamiento psiquiátrico previo. Criterios de exclusión: Evidencia clínica de enfermedad médica descompensada, antecedente de ideación o intento suicida, evidencia clínica de abuso o dependencia a sustancias adictivas. En todos los casos, previo al protocolo de investigación los participantes dieron su consentimiento informado asentando su firma en la carta respectiva (documento elaborado y aprobado por la Comisión de Ética del INPRFM conforme a la Declaración de Helsinki). Después de esto, fueron estudiados durante una sesión de IRMf, donde escucharon dos piezas musicales sin ninguna tarea adicional. 


\section{Procedimiento general}

Las sesiones de neuroimagen se programaron dentro de los tres días siguientes al diagnóstico. Se realizaron en la Unidad de imágenes cerebrales del INPRFM. Para efectuar la sesión con los estímulos musicales los participantes, proveídos con audífonos no-magnéticos (Avotec Inc., Stuart, Fl, EU), fueron introducidos mediante la camilla deslizable integrada al resonador. Se aplicó un diseño experimental en bloques. El tiempo dentro del escáner fue de aproximadamente 30 minutos más el tiempo de adquisición de la referencia anatómica. Las sesiones tuvieron una duración máxima de cuarenta y cinco minutos.

\section{Estímulos}

Durante las sesiones de IRMf se presentaron dos obras de música instrumental previamente validadas por su efecto emocional., $8,15,16$ Una pieza fue de música contemporánea original del compositor Jean Prodromidès (1927- ), para orquesta sinfónica, coros e instrumentos especiales, que induce sentimientos de inquietud. Es una pieza agitada e impredecible con disonancias y efectos impactantes, utilizada como pista musical en la película "Dantón", del cineasta Pruszak, en 1983. La otra pieza fue la "Invención", a tres voces, BWV 797, de Johann Sebastian Bach, una pieza suave y delicada escrita originalmente para clavecín e interpretada al piano por el pianista Glenn Gould, en la grabación de 1959. Esta obra se caracteriza por la inducción probada de sentimientos de tranquilidad. Adicionalmente, como estímulo control, se usó ruido blanco extraído de la banda de FM.

Los estímulos musicales fueron preparados por medio del programa libre de audiograbación y edición, Audacity (http://audacity.sourceforge.net/?lang=es [28/01/13]). Se seleccionó un pasaje de cada pieza musical para completar una duración de cuatro minutos, se dividió en diez bloques iguales de 24 segundos y se intercaló el ruido blanco en bloques de 24 segundos, lo cual resultó en ocho minutos de cada tipo de música y ruido blanco para cada pista. Los estímulos se almacenaron en archivo digital y fueron grabados en un CD para reproducir los estímulos, de forma contrabalanceada, en todas las sesiones.

\section{Resonancia magnética funcional}

La adquisición de las imágenes cerebrales se llevó a cabo en un equipo Philips Achieva 3.0 T (Philips Medical Syste$\mathrm{ms}$, Eindhoven, Holanda). Durante la presentación de cada una de las piezas musicales editadas se obtuvo una serie de imágenes ecoplanares EPI-SingleShot en orientación axial, pesadas a $\mathrm{T} 2 *(\mathrm{TR}=2000 \mathrm{~ms}, \mathrm{TE}=35 \mathrm{~ms})$, en 31 cortes de $4 \mathrm{~mm}$, con tamaño de voxel de $3 \times 3 \times 4 \mathrm{~mm}^{3}$, cubriendo el volumen cerebral completo. Como referencia anatómica de alta resolución y contraste se incluyó la adquisición de imágenes T1 con pulsos de inversión recuperación (IR), $\mathrm{TR}=2949 \mathrm{~ms}$, TE $=15 \mathrm{~ms}$, tiempo de inversión (TI) 400ms, con la misma localización y orientación de las imágenes funcionales.

\section{Análisis de las imágenes}

Los datos fueron transferidos a una estación de trabajo mediante un formato de imagen propio del escáner Philips. Las imágenes fueron transferidas y salvadas dentro del formato de análisis SPM2 como grupos en orden temporal usando el sistema MRIcro de Chris Rorden. Se analizaron estadísticamente con el software SPM2 (Wellcome Department of Imaging Neuroscience). La alineación se aseguró usando los procedimientos estandarizados incluidos en SPM. ${ }^{17}$ Se aplicó el suavizado de las imágenes con núcleo Gaussiano de FWHM de $6 \mathrm{~mm}$. Los signos funcionales se obtuvieron con correlación a la función Box-car y con convolución a la función de la respuesta hemodinámica sin la corrección derivativa de tiempo. ${ }^{17} \mathrm{El}$ análisis estadístico de los datos se basó en el Modelo General Lineal (GLM). Los clusters compuestos por menos de cinco voxels se excluyeron del análisis. La significancia de los mapas de probabilidad se computaron para cada condición dentro del espacio estandarizado de Talairach. ${ }^{18}$

\section{RESULTADOS}

La imagen de las áreas activadas se resume en la figura 1. La lista de máximos locales con valor 1-p para cada grupo de voxeles correspondientes a cada condición se muestran en los cuadros 1-4.

Fueron evidentes las diferencias entre los pacientes con TDM (p/TDM) y los s/C, y también entre las obras de Bach y Prodromidès (figura 1 ). El grupo $\mathrm{p} / \mathrm{TDM}$, presentó el menor número de activaciones para ambos tipos de música. En

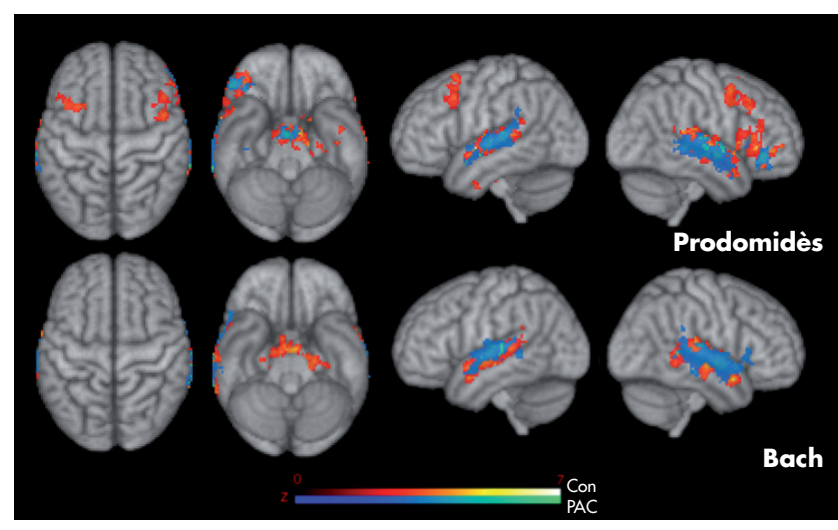

Figura 1. Contraste BOLD en los grupos de pacientes TDM (azulverde) y sujetos control (rojo-amarillo) durante la presentación de la música inquietante (Prodomidès) y la música tranquila (Bach). 
Flores-Gutiérrez et al.

Cuadro 1. Localización anatómica de las áreas activadas en los p/TDM al escuchar la obra de Prodromidès durante el estudio de IRMf

\begin{tabular}{|c|c|c|c|c|c|c|c|c|c|c|c|c|c|}
\hline \multicolumn{14}{|c|}{ Pacientes/Prodromidès } \\
\hline Izquierdo & BA & $X$ & Y & Z & $\begin{array}{l}\text { Rango } \\
(\mathrm{mm})\end{array}$ & $\begin{array}{l}\text { Datos } \\
\text { anexados }\end{array}$ & Derecho & BA & $X$ & Y & Z & $\begin{array}{l}\text { Rango } \\
(\mathrm{mm})\end{array}$ & $\begin{array}{c}\text { Datos } \\
\text { anexados }\end{array}$ \\
\hline Hipocampo & & -33 & -31 & -3 & 1 & 9 & Frontal Medial & 46 & 51 & 48 & 14 & 0 & 14 \\
\hline Hipocampo Sub-Giral & -33 & -29 & -5 & 2 & 9 & & Frontal Medial & 47 & 40 & 35 & -5 & 2 & 14 \\
\hline Hipocampo Sub-Giral & -31 & -27 & -5 & 2 & 9 & & Frontal Medial & 47 & 46 & 39 & -6 & 1 & 14 \\
\hline Hipocampo Sub-Giral & -28 & -25 & -7 & 0 & 4 & & Frontal Medial & 47 & 33 & 35 & -5 & 2 & 1 \\
\hline Cola del núcleo caudado & -35 & -30 & -5 & 2 & 9 & & Frontal Inferior & 47 & 53 & 36 & -6 & 0 & 14 \\
\hline Cola del núcleo caudado & -33 & -33 & -1 & 1 & 5 & & Frontal Inferior & 47 & 50 & 36 & -8 & 1 & 14 \\
\hline Temporal Superior & 41 & -43 & -37 & 9 & 1 & 16 & Frontal Inferior & 47 & 47 & 37 & -12 & 0 & 14 \\
\hline Temporal Superior & 41 & -51 & -34 & 13 & 3 & 16 & Claustrum & & 28 & 8 & 10 & 0 & 15 \\
\hline Temporal Superior & 22 & -61 & -27 & 4 & 0 & 16 & Hipocampo Sub-Giral & & 34 & -30 & -5 & 2 & 13 \\
\hline Temporal Superior & 38 & -50 & 4 & -14 & 1 & 16 & Hipocampo Sub-Giral & & 33 & -28 & -7 & 0 & 13 \\
\hline Temporal Medial & 22 & -62 & -29 & 5 & 1 & 16 & Hipocampo Sub-Giral & & 34 & -26 & -7 & 0 & 13 \\
\hline \multirow[t]{26}{*}{ Temporal Medial } & 21 & -54 & -8 & -9 & 3 & 16 & Hipocampo Sub-Giral & & 32 & -29 & -5 & 1 & 13 \\
\hline & & & & & & & Hipocampo Sub-Giral & & 33 & -31 & -3 & 1 & 13 \\
\hline & & & & & & & Hipocampo Sub-Giral & & 25 & -44 & 5 & 3 & 12 \\
\hline & & & & & & & Hipocampo Sub-Giral & & 23 & -41 & 7 & 4 & 12 \\
\hline & & & & & & & Tálamo & & 13 & -4 & 6 & 0 & 15 \\
\hline & & & & & & & Tálamo & & 3 & -8 & -3 & 3 & 15 \\
\hline & & & & & & & Tálamo Pulvinar & & 12 & -29 & 17 & 2 & 10 \\
\hline & & & & & & & Tálamo Pulvinar & & 13 & -31 & 15 & 2 & 10 \\
\hline & & & & & & & Tálamo Pulvinar & & 16 & -31 & 15 & 2 & 10 \\
\hline & & & & & & & Tálamo Pulvinar & & 18 & -33 & 13 & 1 & 8 \\
\hline & & & & & & & Tálamo Pulvinar & & 18 & -35 & 11 & 2 & 6 \\
\hline & & & & & & & Tálamo Pulvinar & & 14 & -25 & 19 & 2 & 2 \\
\hline & & & & & & & Cola del núcleo caudado & & 33 & -31 & -1 & 1 & 13 \\
\hline & & & & & & & Cola del núcleo caudado & & 21 & -39 & 9 & 3 & 7 \\
\hline & & & & & & & Núcleo Lentiforme & & 13 & -2 & 6 & 1 & 15 \\
\hline & & & & & & & Putamen & & 26 & 6 & 10 & 1 & 15 \\
\hline & & & & & & & Putamen & & 25 & -9 & 11 & 0 & 11 \\
\hline & & & & & & & Putamen & & 26 & -10 & 6 & 0 & 11 \\
\hline & & & & & & & Putamen & & 27 & 8 & 8 & 0 & 15 \\
\hline & & & & & & & Temporal Superior & 42 & 67 & -11 & 6 & 0 & 17 \\
\hline & & & & & & & Temporal Superior & 22 & 67 & -10 & 4 & 1 & 17 \\
\hline & & & & & & & Temporal Superior & 22 & 61 & 1 & -3 & 1 & 17 \\
\hline & & & & & & & Temporal Superior & 22 & 69 & -38 & 5 & 1 & 17 \\
\hline & & & & & & & Temporal Medial & 22 & 70 & -36 & 3 & 2 & 17 \\
\hline & & & & & & & Temporal Medial & 22 & 73 & -35 & 2 & 4 & 17 \\
\hline & & & & & & & Fusiforme & 20 & 45 & -11 & -23 & 1 & 3 \\
\hline
\end{tabular}

BA = Áreas de Brodmann; X, Y, Z = Coordenadas de Talairach: $X([-]$ izquierda; [+] derecha), Y ([-] posterior; [+] anterior), Z ([-] inferior; [+] superior ) (Lancaster et al., 2000). Radio de los "Clusters" $\geq 5$ vóxeles; "Family Wise Error" corregido $p=0.05$ (Friston et al., 1995).

ese sentido la obra de Bach fue la más poco demandante, porque tanto los s/C como los p/TDM mostraron el menor número de activaciones (cuadros 3 y 4 ). Concretamente, sólo tuvieron actividad en el núcleo caudado izquierdo (cola) y en el hemisferio derecho en zonas restringidas del hipocampo, el parahipocampo y el giro fusiforme. Estas dos últimas estuvieron activas en todos los casos. Por el contrario, fue durante la música de Prodromidès donde todos los sujetos reclutaron mucho mayor número de regiones cerebrales (cuadros 1 y 2).

Los ganglios basales tuvieron diferentes activaciones durante la obra de Prodromidès: el núcleo caudado (cuerpo) bilateral sólo en los s/C; también el putamen y el globus palidus se activaron tanto en los s/C como en los p/TDM (cuadros 1 y 2). El núcleo caudado (cola) estuvo activo en todas las condiciones. El claustrum derecho generó un máximo de actividad sólo en los p/TDM durante la escucha del fragmento de Prodromidès (cuadro 1). Esta obra también requirió en los p/TDM actividad de los ganglios basales, además de otras estructuras (cuadro 3 ).

El lóbulo anterior de la ínsula tuvo activación en los s/C tanto para la música tranquila como para la música inquietante, pero en los p/TDM no la hubo para ninguna de las dos obras musicales (cuadros 1 y $3-2$ y 4 ).

La corteza parahipocampal posterior tuvo activaciones sólo en la obra de Bach, siendo bilateral en los s/C y derecha en los p/TDM. La obra de Bach no demandó actividad frontal en ningún grupo (cuadros 3 y 4). En cambio, la obra de Prodromidès sí demandó abundante actividad frontal, bilateral en los s/C, y derecha en los p/TDM (cuadros 3 y 4). 
Cuadro 2. Localización anatómica de las áreas activadas en los $\mathrm{s} / \mathrm{C}$ al escuchar la obra de Prodromidès durante el estudio de IRMf

\begin{tabular}{|c|c|c|c|c|c|c|c|c|c|c|c|c|c|}
\hline \multicolumn{14}{|c|}{ Controles/Prodromidès } \\
\hline Izquierdo & BA & $X$ & Y & Z & $\begin{array}{l}\text { Rango } \\
(\mathrm{mm})\end{array}$ & $\begin{array}{c}\text { Datos } \\
\text { anexados }\end{array}$ & Derecho & BA & $X$ & Y & Z & $\begin{array}{l}\text { Rango } \\
(\mathrm{mm})\end{array}$ & $\begin{array}{l}\text { Datos } \\
\text { anexados }\end{array}$ \\
\hline Frontal Superior & 8 & -43 & 17 & 55 & 3 & 8 & Frontal Medial & 6 & 42 & 8 & 54 & 0 & 26 \\
\hline Frontal Medial & 6 & -25 & 16 & 58 & 1 & 25 & Frontal Medial & 6 & 33 & 17 & 63 & 5 & 18 \\
\hline Frontal Medial & 6 & -27 & 15 & 58 & 1 & 25 & Frontal Medial & 6 & 35 & 15 & 62 & 4 & 18 \\
\hline Frontal Medial & 8 & -43 & 27 & 49 & 2 & 15 & Frontal Medial & 6 & 45 & 7 & 52 & 1 & 26 \\
\hline Frontal Medial & 8 & -47 & 29 & 41 & 1 & 7 & Frontal Medial & 6 & 44 & 12 & 52 & 0 & 26 \\
\hline Frontal Precentral & 9 & -38 & 16 & 35 & 0 & 25 & Frontal Medial & 8 & 55 & 17 & 41 & 1 & 6 \\
\hline Frontal Precentral & 9 & -43 & 18 & 34 & 0 & 25 & Frontal Medial & 8 & 55 & 15 & 41 & 0 & 5 \\
\hline Frontal Precentral & 9 & -42 & 19 & 40 & 3 & 25 & Frontal Medial & 8 & 36 & 16 & 45 & 2 & 26 \\
\hline Frontal Precentral & 9 & -40 & 16 & 40 & 3 & 25 & Frontal Medial & 9 & 47 & 7 & 38 & 1 & 26 \\
\hline Postcentral & 40 & -67 & -20 & 16 & 2 & 9 & Frontal Medial & 11 & 41 & 36 & -14 & 1 & 27 \\
\hline Cíngulo Anterior & 32 & -10 & 22 & 38 & 0 & 21 & Frontal Medial & 47 & 41 & 36 & -10 & 1 & 27 \\
\hline Cíngulo Anterior & 32 & -13 & 23 & 37 & 0 & 21 & Frontal Inferior & 10 & 40 & 46 & -2 & 2 & 27 \\
\hline Cíngulo Anterior & 32 & -13 & 19 & 39 & 2 & 21 & Frontal Inferior & 46 & 37 & 35 & 12 & 0 & 27 \\
\hline Cíngulo Anterior & 32 & -21 & 7 & 45 & 1 & 19 & Frontal Inferior & 47 & 42 & 31 & 0 & 5 & 27 \\
\hline Sub-Giral & 32 & -11 & 23 & 40 & 0 & 21 & Precentral & 9 & 40 & 23 & 36 & 0 & 26 \\
\hline Ínsula & 13 & -31 & -30 & 17 & 1 & 28 & Tálamo & & 21 & -20 & 13 & 0 & 11 \\
\hline Ínsula & 13 & -33 & -19 & 21 & 0 & 12 & Cuerpo del Caudado & & 13 & -4 & 25 & 4 & 13 \\
\hline Tálamo & & -7 & -4 & 3 & 0 & 29 & Cíngulo Posterior & 23 & 9 & -29 & 27 & 2 & 10 \\
\hline Tálamo & & -7 & -3 & 1 & 2 & 29 & Temporal Superior & 42 & 70 & -21 & 11 & 1 & 30 \\
\hline Tálamo & & -3 & -4 & 3 & 1 & 29 & Temporal Superior & 42 & 70 & -23 & 12 & 1 & 30 \\
\hline Cola del núcleo caudado & & -37 & -31 & -7 & 3 & 24 & Temporal Superior & 22 & 67 & -1 & 2 & 1 & 30 \\
\hline Cola del núcleo caudado & & -31 & -35 & 1 & 2 & 3 & Temporal Superior & 22 & 61 & -1 & 2 & 0 & 30 \\
\hline Cuerpo del Caudado & & -19 & 7 & 21 & 4 & 20 & Temporal Superior & 22 & 61 & -2 & 4 & 1 & 30 \\
\hline Cuerpo del Caudado & & -17 & 7 & 21 & 2 & 20 & Temporal Superior & 22 & 65 & -1 & 5 & 0 & 30 \\
\hline Cuerpo del Caudado & & -18 & -1 & 17 & 4 & 4 & Temporal Superior & 22 & 69 & -47 & 18 & 1 & 16 \\
\hline Núcleo Lentiforme & & -6 & -2 & -1 & 2 & 29 & Temporal Superior & 22 & 69 & -44 & 16 & 1 & 16 \\
\hline Putamen & & -17 & 7 & -5 & 0 & 29 & Temporal Superior & 22 & 68 & -43 & 10 & 0 & 23 \\
\hline Parahipocampal & 34 & -10 & -6 & -17 & 3 & 29 & Temporal Superior & 22 & 71 & -36 & 9 & 2 & 23 \\
\hline Hipocampo Sub-Giral & & -34 & -28 & -8 & 1 & 24 & Temporal Medial & 22 & 73 & -35 & 3 & 4 & 23 \\
\hline Hipocampo Sub-Giral & & -35 & -31 & -7 & 3 & 24 & Temporal Medial & 22 & 67 & -42 & 5 & 0 & 23 \\
\hline Temporal Transverso & 41 & -37 & -34 & 13 & 0 & 28 & Temporal Medial & 22 & 69 & -41 & 4 & 1 & 23 \\
\hline Temporal Transverso & 42 & -61 & -11 & 8 & 2 & 28 & Temporal Medial & 21 & 71 & -35 & -5 & 3 & 2 \\
\hline Temporal Superior & 22 & -67 & -12 & 1 & 2 & 28 & Temporal Medial & 21 & 71 & -41 & -13 & 4 & 1 \\
\hline Temporal Superior & 22 & -52 & -3 & -3 & 1 & 28 & Temporal Medial & 21 & 71 & -19 & -13 & 3 & 17 \\
\hline Temporal Superior & 22 & -69 & -19 & 1 & 1 & 28 & Temporal Medial & 21 & 69 & -43 & -15 & 5 & 14 \\
\hline Temporal Medial & 21 & -44 & -2 & -29 & 0 & 22 & Temporal Medial & 21 & 70 & -37 & 3 & 2 & 23 \\
\hline Temporal Inferior & 20 & -43 & -14 & -27 & 1 & 24 & & & & & & & \\
\hline Temporal Inferior & 20 & -46 & -4 & -37 & 3 & 22 & & & & & & & \\
\hline Temporal Inferior & 20 & -46 & -3 & -35 & 1 & 22 & & & & & & & \\
\hline Temporal Inferior & 20 & -45 & -6 & -28 & 1 & 22 & & & & & & & \\
\hline Temporal Inferior & 20 & -45 & -4 & -29 & 0 & 22 & & & & & & & \\
\hline Temporal Inferior & 20 & -44 & -8 & -29 & 1 & 22 & & & & & & & \\
\hline Fusiforme & 20 & -42 & -12 & -25 & 1 & 24 & & & & & & & \\
\hline Fusiforme & 20 & -42 & -13 & -23 & 0 & 24 & & & & & & & \\
\hline
\end{tabular}

$\mathrm{BA}=$ Áreas de Brodmann; $X, Y, Z=$ Coordenadas de Talairach: $X([-]$ izquierda; $[+]$ derecha), $Y([-]$ posterior; $[+]$ anterior), Z ([-] inferior; [+] superior ) (Lancaster et al., 2000). Radio de los "Clusters" $\geq 5$ vóxeles; "Family Wise Error" corregido $p=0.05$ (Friston et al., 1995).

\section{DISCUSIÓN}

Este estudio compara la respuesta metabólica cerebral de pacientes masculinos con trastorno depresivo mayor en su primer episodio y sin ningún tratamiento contra la de sujetos control, con la única tarea de escuchar dos tipos de música desconocida para los participantes (una tranquila y otra inquietante). Confirmamos la hipótesis de que los p/TDM tendrían hipofunción en áreas relacionadas con el procesa- miento neurocognitivo de la música, a diferencia de los s/C quienes mostraron actividad en áreas cerebrales típicas para este tipo de estímulo. ${ }^{19-21}$

\section{Comparación con otras publicaciones}

Existen escasos trabajos que relacionan el TDM con la experiencia musical, solamente encontramos tres estudios conductuales y sólo uno donde se aplica la técnica de IRMf. 
Cuadro 3. Localización anatómica de las áreas activadas en los p/TDM al escuchar la obra de Bach durante el estudio de IRMf

\begin{tabular}{|c|c|c|c|c|c|c|c|c|c|c|c|c|c|}
\hline \multicolumn{14}{|c|}{ Pacientes/Bach } \\
\hline Izquierdo & BA & $x$ & Y & Z & $\begin{array}{l}\text { Rango } \\
(\mathrm{mm})\end{array}$ & $\begin{array}{l}\text { Datos } \\
\text { anexados }\end{array}$ & Derecho & BA & $x$ & Y & Z & $\begin{array}{l}\text { Rango } \\
(\mathrm{mm})\end{array}$ & $\begin{array}{c}\text { Datos } \\
\text { anexados }\end{array}$ \\
\hline Cola del núcleo caudado & & -37 & -31 & -7 & 3 & 8 & Parahipocampal & 28 & 26 & -23 & -10 & 2 & 7 \\
\hline Cola del núcleo caudado & & -35 & -29 & -5 & 1 & 8 & Hipocampo Sub-Giral & & 27 & -24 & -8 & 3 & 7 \\
\hline Temporal Superior & 42 & -64 & -26 & 9 & 1 & 9 & Hipocampo Sub-Giral & & 29 & -25 & -5 & 2 & 7 \\
\hline Temporal Superior & 41 & -43 & -36 & 3 & 3 & 5 & Temporal Superior & 42 & 71 & -29 & 19 & 3 & 6 \\
\hline Temporal Superior & 22 & -61 & -25 & 7 & 2 & 9 & Temporal Superior & 42 & 68 & -28 & 7 & 0 & 10 \\
\hline Temporal Superior & 22 & -53 & -12 & -2 & 0 & 9 & Temporal Superior & 42 & 67 & -28 & 9 & 0 & 10 \\
\hline Temporal Superior & 22 & -59 & -26 & 6 & 1 & 9 & Temporal Superior & 22 & 68 & -40 & 7 & 0 & 10 \\
\hline Temporal Medial & 22 & -55 & -34 & 6 & 1 & 9 & Temporal Superior & 22 & 66 & -40 & 7 & 1 & 10 \\
\hline \multirow[t]{6}{*}{ Temporal Medial } & 21 & -55 & -19 & -2 & 2 & 9 & Temporal Medial & 21 & 71 & -25 & -5 & 3 & 10 \\
\hline & & & & & & & Temporal Medial & 21 & 63 & 4 & -9 & 1 & 10 \\
\hline & & & & & & & Temporal Medial & 21 & 71 & -30 & -10 & 3 & 4 \\
\hline & & & & & & & Fusiforme & 20 & 41 & -9 & -23 & 1 & 2 \\
\hline & & & & & & & Fusiforme & 20 & 45 & -11 & -23 & 1 & 1 \\
\hline & & & & & & & Sub-Giral & 20 & 43 & -13 & -21 & 1 & 3 \\
\hline
\end{tabular}

$\mathrm{BA}=$ Áreas de Brodmann; $\mathrm{X}, \mathrm{Y}, \mathrm{Z}=$ Coordenadas de Talairach: $\mathrm{X}([-]$ izquierda; $[+]$ derecha), $\mathrm{Y}([-]$ posterior; $[+]$ anterior), $Z([-]$ inferior; $[+]$ superior $)($ Lancaster et al., 2000). Radio de los "Clusters" $\geq 5$ vóxeles; "Family Wise Error" corregido $p=0.05$ (Friston et al., 1995).

Los estudios conductuales son los de Nielzèn, ${ }^{4}$ Punkanen $^{5}$ y Naranjo. ${ }^{6}$ Los principales hallazgos de esos estudios fueron: Primero, al igual que en nuestro trabajo los pacientes con depresión muestran menos reactividad a la música; y segundo, se confirma en pacientes con TDM un sesgo negativo en las emociones y el reconocimiento de estímulos interpersonales que también afectan a la forma de percibir la música. Nuestro trabajo es congruente con esos reportes, y sabemos que la hipoactivación del estriado ventral, evidente en nuestros p/TDM, puede llevar a una disminución del placer en la experiencia musical, expresada como poca reactividad emocional e indiferencia. Por otra parte, el

Cuadro 4. Localización anatómica de las áreas activadas en los $\mathrm{s} / \mathrm{C}$ al escuchar la obra de Bach durante el estudio de IRMf

\begin{tabular}{|c|c|c|c|c|c|c|c|c|c|c|c|c|c|}
\hline \multicolumn{14}{|c|}{ Controles/Bach } \\
\hline Izquierdo & BA & $X$ & Y & Z & $\begin{array}{l}\text { Rango } \\
(\mathrm{mm})\end{array}$ & $\begin{array}{c}\text { Datos } \\
\text { anexados }\end{array}$ & Derecho & BA & $\mathrm{X}$ & Y & Z & $\begin{array}{l}\text { Rango } \\
(\mathrm{mm})\end{array}$ & $\begin{array}{l}\text { Datos } \\
\text { anexados }\end{array}$ \\
\hline Insula & 13 & -53 & -38 & 15 & 3 & 11 & Insula & 13 & 39 & -22 & -9 & 3 & 6 \\
\hline Área Parahipocampal & 30 & -18 & -37 & 4 & 1 & 10 & Área Parahipocampal & 19 & 31 & -41 & -5 & 1 & 9 \\
\hline Área Parahipocampal & 28 & -16 & -20 & -17 & 3 & 10 & Área Parahipocampal & 37 & 34 & -43 & -8 & 0 & 9 \\
\hline Tálamo & & -3 & -10 & -3 & 3 & 10 & Área Parahipocampal & 36 & 39 & -33 & -7 & 4 & 8 \\
\hline Tálamo & & -1 & -9 & 0 & 2 & 10 & Área Parahipocampal & 36 & 39 & -21 & -11 & 4 & 2 \\
\hline Sub-Giral & & -3 & -41 & -3 & 2 & 10 & Área Parahipocampal & 36 & 41 & -21 & -13 & 2 & 1 \\
\hline Temporal Superior & 41 & -35 & -34 & 8 & 2 & 11 & Tálamo & & 1 & -9 & -1 & 2 & 10 \\
\hline Temporal Superior & 22 & -50 & -29 & 8 & 1 & 11 & Cola del núcleo caudado & & 37 & -33 & -4 & 2 & 8 \\
\hline Temporal Superior & 22 & -57 & -35 & -2 & 1 & 11 & Cíngulo Posterior & 29 & 8 & -41 & 10 & 2 & 7 \\
\hline Temporal Superior & 22 & -53 & -10 & 11 & 2 & 11 & Cíngulo Posterior & 29 & 5 & -41 & 10 & 3 & 7 \\
\hline Temporal Medial & 21 & -50 & -38 & -2 & 1 & 11 & Temporal Transverso & 42 & 68 & -17 & 11 & 1 & 12 \\
\hline \multirow[t]{11}{*}{ Cerebelar Lingual } & & -55 & -21 & -7 & 0 & 4 & Temporal Superior & 41 & 57 & -26 & 12 & 1 & 12 \\
\hline & & & & & & & Temporal Superior & 22 & 56 & -11 & 0 & 1 & 12 \\
\hline & & & & & & & Temporal Superior & 42 & 59 & -26 & 14 & 0 & 12 \\
\hline & & & & & & & Temporal Superior & 38 & 50 & 9 & -20 & 0 & 12 \\
\hline & & & & & & & Temporal Medial & 21 & 50 & 1 & -10 & 1 & 12 \\
\hline & & & & & & & Temporal Medial & 21 & 73 & -35 & -5 & 4 & 5 \\
\hline & & & & & & & Temporal Medial & 21 & 67 & -1 & -11 & 1 & 3 \\
\hline & & & & & & & Fusiforme & 37 & 40 & -44 & -11 & 2 & 9 \\
\hline & & & & & & & Fusiforme & 37 & 42 & -44 & -11 & 4 & 9 \\
\hline & & & & & & & Fusiforme & 37 & 43 & -42 & -13 & 2 & 9 \\
\hline & & & & & & & Fusiforme & 37 & 37 & -44 & -10 & 1 & 9 \\
\hline
\end{tabular}

BA = Áreas de Brodmann; $X, Y, Z=$ Coordenadas de Talairach: $X([-]$ izquierda; $[+]$ derecha), $Y([-]$ posterior; $[+]$ anterior $), Z$ ([-] inferior; [+] superior ) (Lancaster et al., 2000). Radio de los "Clusters" $\geq 5$ vóxeles; "Family Wise Error" corregido $p=0.05$ (Friston et al., 1995). 
circuito fronto-estriatal, que se ha relacionado con la anticipación musical ${ }^{22}$ y el circuito del placer, ${ }^{23}$ parece indispensable en la función gratificante para dar sentido a la escucha musical. ${ }^{24}$ En cuanto al sesgo hacia emociones y pensamientos negativos, presente en los pacientes deprimidos, ${ }^{25,26}$ éste podría corresponder al exceso de activación de la Default Mode Network (DMN) ${ }^{27}$ que ya ha sido reportada hiperactiva en pacientes con depresión. ${ }^{28-30}$ En la DMN se ha propuesto a la corteza cingulada posterior como responsable de los pensamientos autoreferenciales con sesgo negativo. ${ }^{30}$ Nuestros resultados son congruentes con la propuesta del efecto de interferencia cognitiva y afectiva que produce la DMN. ${ }^{29,31}$ En nuestros p/TDM las imágenes muestran ausencia o disminución de la actividad en estructuras mediales, estriatales, límbicas y para-límbicas, durante la escucha de música.

Por último, el estudio de Osuch ${ }^{7}$ contrastó la escucha de diferentes tipos de música favorita contra música neutra en pacientes deprimidos y controles usando la técnica de IRMf. Su propósito fue investigar la activación del circuito de recompensa. Encontraron activación del circuito en los sujetos control, pero no así en los pacientes con depresión, en quienes sólo encontraron el giro parahipocampal izquierdo correlacionado con la música agradable. Nuestros resultados coinciden en cuanto a la baja respuesta de regiones activadas en los p/TDM a diferencia de los controles, pero también encontramos el mismo fenómeno en el giro parahipocampal en los p/TDM con la música agradable, sólo que en hemisferio derecho (BA 28). El giro parahipocampal ya ha sido relacionado con la escucha musical; algunas investigaciones lo implican en una contribución clave hacia tareas de recuperación de memoria y aprendizaje, particularmente en la memoria contextual y también en el procesamiento de la novedad. ${ }^{32,33}$ Es posible que la diferencia de lateralidad hemisférica entre el reporte de $\mathrm{Osuch}^{7}$ y el nuestro esté dada porque ellos usaron piezas musicales muy familiares y favoritas de cada sujeto, por lo cual el procesamiento fue predominante en el hemisferio izquierdo; en cambio, en nuestro estudio las obras fueron nuevas y desconocidas para los participantes y posiblemente por ello produjeron un procesamiento de predominio frontotemporal derecho, como ya se ha reportado en otros estudios. $^{34}$

\section{Diferencias entre pacientes y controles}

En la comparación de los dos tipos de música todos los sujetos reclutaron un mayor número de regiones cerebrales al escuchar la obra de Prodromidès. Este efecto se ha reportado previamente ${ }^{16} \mathrm{y}$ posiblemente implica una incorporación neuronal más abundante en la búsqueda de significado para una obra de estructura y forma poco ortodoxa, enérgica e impredecible. Por el contrario, en la obra de Bach todos los sujetos mostraron el menor número de activaciones, posi- blemente como una respuesta de economía ante un estímulo familiar con estructura y forma predecible cuyo sustrato son las redes neuronales preestablecidas por aprendizaje o por conocimiento implícito. ${ }^{35-37}$ Algunos autores consideran que ese puede ser un fenómeno de enculturación, por exposición a un lenguaje musical tonal desde la infancia, que incorpora reglas musicales (gramaticales, sintácticas, etc.) que llegan a ser tan familiares como las del lenguaje materno. ${ }^{38}$ De ese modo el oyente, familiarizado con tales formas, desarrolla expectativas de los acontecimientos musicales y una facilitación para las secuencias que se van generando momento a momento. ${ }^{36,39-41}$

\section{Otras regiones activadas}

La obra de Prodromidès generó actividad en el claustrum derecho de los pacientes con TDM. Esta delgada estructura laminar, ubicada entre el putamen y el lóbulo de la ínsula, cuenta con neuronas multimodales con conexiones distantes cuya función, hasta donde se sabe, es coordinar regiones corticales motoras con la adquisición de información sensorial en el reconocimiento perceptual del ambiente que nos rodea, posiblemente para sincronizar acciones relacionadas con la orientación espacial y la atención dirigida. ${ }^{42}$ En la experiencia de la música y, en general, de los sonidos, la sincronización de eventos, la orientación espacial y la atención dirigida son particularmente importantes, tanto para procesar el significado sonoro como en la ejecución motora subsecuente. Además, se ha observado que la actividad del claustrum es requerida en situaciones relevantes de auto-conservación. La música de Prodromidès logra una estimulación generadora de estrés con sonidos agresivos y amenazantes que promueven respuestas de sobrevivencia con tendencias psicomotoras hacia la acción, que necesariamente implicarían a dicha estructura. ${ }^{42-45}$

En los s/C la ínsula anterior se activó tanto para la música agradable como para la desagradable, pero en los $\mathrm{p} /$ TDM no aparece activada la ínsula en ninguna de las dos obras presentadas. Esto es congruente con algunas publicaciones que reportan disminución de la respuesta del lóbulo anterior de la ínsula en pacientes con depresión unipolar. ${ }^{46-48}$ Por otra parte, a la ínsula también se le ha relacionado con las emociones sociale ${ }^{49} \mathrm{y}$ con la función de procesador paralímbico multimodal. ${ }^{50}$ En la experiencia musical se le ha relacionado con el procesamiento gramatical, emocional, cognitivo y rítmico. ${ }^{51-53}$ En nuestro trabajo encontramos que, a diferencia de los s/C, en los p/TDM el lóbulo anterior de la ínsula no muestra actividad. Es posible que la disminución o ausencia de la actividad estriatal, límbica e hipotalámica en los p/TDM tengan una posible repercusión visceral que disminuya la información de retroalimentación emocional interoceptiva propia de la ínsula.

Por último, los ganglios basales también tuvieron diferentes activaciones: la cola del núcleo caudado estuvo 
presente en todos los casos, el cuerpo del núcleo caudado sólo en los s/C durante la obra de Prodromidès que también activó el putamen de los s/C y de los p/TDM. Sabemos que los ganglios basales son indispensables en la expresión de esquemas correctos de acción y en la selección apropiada de respuestas motoras, incluyendo las de los estados emocionales y sociales. Estos procesos son fundamentales para toda acción dirigida a metas. ${ }^{54}$ En nuestro estudio estas activaciones en los participantes al oír la música, principalmente en la cola del núcleo caudado, nos hablan de una intención o una acción, desde marcar el tempo usando un dedo o un pié, hasta querer alejarse o detener la música inquietante. La cola del núcleo caudado guarda una estrecha relación con las funciones amigdalinas y tiene notable influencia en las demás estructuras de los ganglios basales..$^{54}$ También recibe conexiones de áreas témporo-parietales e, indirectamente, de la corteza frontal. ${ }^{55,56}$ Además, se le ha relacionado con funciones cognitivas como el aprendizaje y la planeación de conductas. ${ }^{57,58}$ Dado que las obras no eran conocidas por los participantes, es posible que en la cola del núcleo caudado también se haya registrado actividad como parte de la experiencia de aprendizaje. ${ }^{57}$

\section{CONCLUSIÓN}

Nuestros resultados muestran diferencias relacionadas con el tipo de música y el tipo de participante (paciente depresivo o sujeto control). En todos los casos fueron activados el giro temporal superior y medial debido al procesamiento sensorial auditivo, también el giro fusiforme, regiones parahipocámpicas y el nucleo caudado (cola), que tuvieron activación en todas las condiciones. La mayor demanda cortical que incluyó regiones frontales fue registrada en los $\mathrm{s} / \mathrm{C}$ con la música inquietante. El grupo de p/TDM presentó el menor número de regiones cerebrales activadas para ambos tipos de música y más para la obra validada como tranquila, lo cual podría interpretarse como el procesamiento musical mínimo, en las condiciones de alteración por el TDM de este estudio. Los resultados en los p/TDM también son congruentes con otros reportes que muestran hipofunción de la corteza frontal, los ganglios basales, el sistema límbico y otras zonas paralímbicas. ${ }^{59-62}$ Son necesarias nuevas investigaciones para ampliar la experimentación con música ya que puede aportar información relevante acerca del TDM.

\section{AGRADECIMIENTOS}

Este trabajo se realizó gracias a un apoyo para la investigación de la Dirección de Servicios Clínicos del Instituto Nacional de Psiquiatría Ramón de la Fuente Muñiz.

Agradecemos a los doctores María Corsi Cabrera y José Luis Díaz Gómez su constante apoyo y la revisión del manuscrito.

\section{REFERENCIAS}

1. Lara-Muñoz MC, Robles-García R, Orozco R, Real T et al. Estudio de costo-efectividad del tratamiento de la depresión en México. Salud Mental 2010;33:301-308.

2. Ruiz LG, Colin RF, Corlay IS, Lara MC et al. Trastorno depresivo mayor en México: La relación entre la intensidad de la depresión, los síntomas físicos dolorosos y la calidad de vida. Salud Mental 2007;30(02):25-32.

3. Medina-Mora ME, Borges G, Benjet C, Lara C et al. Psychiatric disorders in Mexico: lifetime prevalence in a nationally representative sample. British J Psychiatry 2007;190:521-528.

4. Nielzén $S$, Cesarec Z. Emotional experience of music by psychiatric patients, compared with normal subjects. Acta Psychiatrica Scandinavica 1982;65(6):450-460.

5. Punkanen M, Eerola T, Erkkilä J. Biased emotional recognition in depression: Perception of emotions in music by depressed patients. J Affect Disord 2011;130(1-2):118-126.

6. Naranjo C, Kornreich C, Campanella S, Noël X et al. Major depression is associated with impaired processing of emotion in music as well as in facial and vocal stimuli. J Affect Disord 2011;28(3):243-51.

7. Osuch EA, Bluhm RL, Williamson PC, Théberge J et al. Brain activation to favorite music in healthy controls and depressed patients. Neuroreport 2009;20(13):1204-1208.

8. Flores-Gutiérrez EO, Díaz JL. The emotional response to music: attribution of emotion words to musical segments. Salud Mental 2009;32(1):21-34.

9. Stefanescu C, Ciobica A. The relevance of oxidative stress status in first episode and recurrent depression. J Affect Disord 2012;143(1-3):34-38.

10. Lee RS, Hermens DF, Porter MA, Redoblado-Hodge MA. A metaanalysis of cognitive deficits in first-episode major depressive disorder. J Affect Disord 2012;140(2):113-124.

11. Guo W, Liu F, Dai $Y$, Jiang $M$ et al. Decreased interhemispheric resting-state functional connectivity in first-episode, drug-naive major depressive disorder. Prog Neuropsychopharmacol Biol Psychiatry 2013;5(41C):24-29.

12. Wang $Y$, Jia $Y$, Chen $X$, Ling $X$ et al. Hippocampal N-acetylaspartate and morning cortisol levels in drug-naive, first-episode patients with major depressive disorder: effects of treatment. J Psychopharmacol 2012;26(11):1463-470.

13. Bschor $T$, Uhr M, Baethge $C$, Lewitzka $U$ et al. Acute antidepressive efficacy of lithium monotherapy, not citalopram, depends on recurrent course of depression. J Clin Psychopharmacol 2013;33(1):38-44.

14. Solis-Ortiz S, Ramos J, Arce C, Guevara MA et al. EEG oscillations during menstrual cycle. Int J Neurosci 1994;76:279-292.

15. Ramos-Loyo J, Guevara MA, Martínez A, Arce C et al. Evaluacion de los estados afectivos provocados por la musica. Revista Mexicana Psicología 1996;13(2):131-145.

16. Flores-Gutiérrez EO, Díaz JL, Barrios FA, Favila-Humara R et al. Metabolic and electric brain patterns during pleasant and unpleasant emotions induced by music masterpieces. International J Psychophysiology 2007;65(1):69-84.

17. Friston KJ, Holmes AP, Poline JB, Grasby PJ et al. Analysis of fMRI time-series revisited. NeuroImage 1995;2:45-53.

18. Lancaster JL, Woldorff MG, Parsons LM, Liotti M, Automated Talairach Atlas labels for functional brain mapping. Hum Brain Mapp 2000;10:120-131.

19. Blood A, Zatorre R. Intensely pleasurable responses to music correlate with activity in brain regions implicated in reward and emotion. Proceedings National Academy Sciences 2001;98(20):11818-11823.

20. Brown S, Martinez MJ, Parsons LM. Passive music listening spontaneously engages limbic and paralimbic systems. Neuroreport 2004;15(13):2033-2037.

21. Koelsch S, Fritz TV, Cramon DY, Muller K et al. Investigating emotion with music: An fMRI study. Human Brain Mapping 2006;27: 239-250. 
22. Leaver Amber M, Jennifer Van Lare, Brandon Zielinski, Andrea R. Brain activation during anticipation of sound sequences. J Neuroscience 2009;29(8):2477-2485.

23. Stoy M, Schlagenhauf F, Sterzer P, Bermpohl F et al. Hyporeactivity of ventral striatum towards incentive stimuli in unmedicated depressed patients normalizes after treatment with escitalopram. J Psychopharmacol 2012;26(5):677-688.

24. Huron D. Sweet anticipation: Music and the psychology of expectation. Cambridge, MA: MIT Press, 2006; Pp. 1-18.

25. Pyszczynski T, Holt K, Greenberg J. Depression, self-focused attention, and expectancies for positive and negative future life events for self and others. J Pers Soc Psychol 1987;52(5):994-1001.

26. Rood L, Roelofs J, Bögels SM, Alloy LB. Dimensions of negative thinking and the relations with symptoms of depression and anxiety in children and adolescents. Cogn Ther Res 2010;34:333-342.

27. Raichle ME, MacLeod AM, Snyder AZ, Powers WJ et al. A default mode of brain function. PNAS 2001;98(2):676-682.

28. Sheline YI, Barcha DM, Price JL, Rundle MM. The default mode network and self-referential processes in depression. PNAS 2009;106(6):1942-1947.

29. Whitfield-Gabrieli S, Ford JM. Default mode network activity and connectivity in psychopathology. Annu Rev Clin Psychol 2012;8:49-76.

30. Berman MG, Peltier S, Nee DE, Kross E et al. Depression rumination and the default network. Soc Cogn Affect Neurosci 2011;6(5):548-555.

31. Sheline YI, Price J, Yan Z, Mintun MA. Resting-state functional MRI in depression unmasks increased connectivity between networks via the dorsal nexus. PNAS 2010;107(24):11020-11025.

32. Hasselmo ME, Stern CE. Mechanisms underlying working memory for novel information. Trends Cogn Sci 2006;10:487-493.

33. Henke K. A model for memory systems based on processing modes rather than consciousness. Nat Rev Neurosci 2010;11:523-532.

34. Altenmüller E, Schürmann K, Lim VK, Parlitz D. Hits to the left, flops to the right: different emotions during listening to music are reflected in cortical lateralization patterns. Neuropsychologia 2002;40(13):2242-2256.

35. Bullmore E, Sporns O. The economy of brain network organization. Nature Reviews 2012;13:336-349.

36. Tillmann B, Bharucha J, Bigand E. Implicit learning of regularities in Western tonal music by self-organization. En: Connectionist models of learning, development and evolution. Proceedings of the sixth neural computation and psychology conference. Londres: Springer; 2001; pp. 175-184.

37. Jones MR, Boltz M. Dynamic attending and responses to time. Psychological Review 1989;96:459-491.

38. Koelsch S, Gunter T, Friederici AD. Brain indices of music processing: "nonmusicians" are musical. J Cognitive Neuroscience 2000;12(3):520541.

39. Bigand E. The influence of implicit harmony, rhythm and musical training on the abstraction of "tension-relaxation schemes" in a tonal musical phrase. Contemporary Music Review 1993;9:128-139.

40. Bigand E. Perceiving musical stability: The effect of tonal structure, rhythm and musical expertise. J Experimental Psychology: Human Perception Performance 1997:21:808-822.

41. Bigand E, Madurell F, Tillmann B, Pineau M. Effect of global structure and temporal organization on chord processing. J Experimental Psychology: Human Perception Performance 1999;25:184-197.
42. Smith JB, Alloway KD. Functional specificity of claustrum connections in the rat: Interhemispheric communication between specific parts of motor cortex. J Neurosci 2010;30(50):16832-16844.

43. Arnow BA, Desmond JE, Banner LL, Glover GH et al. Brain activation and sexual arousal in healthy, heterosexual males. Brain 2002;125:1014-1023.

44. Crick FC, Koch C. What is the function of the claustrum? Phil Trans $R$ Soc B 2005;360:1271-1279.

45. Mathur BN, Caprioli RM, Deutch AY. Proteomic analysis illuminates a novel structural definition of the claustrum and insula. Cerebral Cortex 2009;19:2372-2379.

46. Biver F, Wikler D, Lotstra F, Damhaut $P$ et al. Serotonin 5-HT2 receptor imaging in major depression; focal changes in orbito-insular cortex. Br J Psychiatry 1997;171:444-448.

47. Mayberg HS, Liotti M, Brannan SK, McGinnis S et al. Reciprocal limbic-cortical function and negative mood: converging PET findings in depression and normal sadness. Am J Psychiatry 1999;156:675-682.

48. Kennedy SH, Evans KR, Kruger S, Mayberg HS et al. Changes in regional brain glucose metabolism measured with positron emission tomography after paroxetine treatment of major depression. Am J Psychiatry 2001;158:899-905.

49. Lamm C, Singer T. The role of anterior insular cortex in social emotions. Brain Struct Funct 2010;214:579-591.

50. Nagai M, Kishi K, Kato S. Insular cortex and neuropsychiatric disorders: A review of recent literature. European Psychiatry 2007;22(6):387-394.

51. Patel AD. Language, music, syntax and the brain. Nature Neurosci 2003;6:674-681.

52. Levitin DJ, Menon V. Musical structure is processed in "language" areas of the brain: a possible role for Brodmann Area 47 in temporal coherence. NeuroImage 2003;20:2142-2152.

53. Koelsch S, Kasper E, Gunter TC, Sammler D et al. Music, language, and meaning: Brain signatures of semantic processing. Nat Neurosci 2004;7:302-307.

54. Joseph R. Neuropsychiatry, neuropsychology, clinical neuroscience. New York: Academic Press; 2000.

55. Yeterian EH, Van Hoesen GW. Cortico-striate projections in the rhesus monkey: The organization of certain cortico-caudate connections. Brain Research 1978;139(1):43-63.

56. Lidaka T, Matsumoto A, Ozaki N, Suzuki T et al. Iwata Volume of left amygdala subregion predicted temperamental trait of harm avoidance in female young subjects. A voxel-based morphometry study. Brain Res 2006;1125(1):85-93.

57. Seger CA, Cincotta CM. The roles of the caudate nucleus in human classification learning. J Neuroscience 2005;25(11):2941-2951.

58. Grahn JA, Parkinson JA, Owen AM. The cognitive functions of the caudate nucleus. Prog Neurobiol 2008;86(3):141-55.

59. Nestler E, Barrot M, DiLeone R, Eisch A et al. Neurobiology of depression. Neuron 2002;34:13-25.

60. Galynker I, Cai J, Ongseng F, Finestone $\mathrm{H}$ et al. Hypofrontality and negative symptoms in major depressive disorder. J Nuclear Medicine 1998;39(4):608-612.

61. Ito $H$, Kawashima R, Awata S, Ono $S$ et al. Hypoperfusion in the Limbic System and Prefrontal Cortex in Depression: SPECT with anatomic standardization technique. J Nuclear Medicine 1996;37(3):410-414.

62. Mayberg H, Lewis $P$, Regenold W, Wagner H Jr. Paralimbic hypoperfusion in unipolar depression. J Nuclear Medicine 1994;35(6);929-934.

Artículo sin conflicto de intereses 